\title{
An air circulation purifying system based on PLC
}

\author{
Yin Chen ${ }^{1}$, Chengliang Han, Peng Chen, Zong Chen, L Y Liu and T Du ${ }^{1, *}$ \\ ${ }^{1}$ School of Metallurgy, Northeastern University, Shenyang, Liaoning Province, China
}

\begin{abstract}
Existing air purifiers are mostly indoor air circulation, and most of them cannot achieve indoor and outdoor air circulation and provide fresh air to room. At the same time, the current air purifier filter on the market after absorbing a large amount of dust and other pollutants, if not replaced in time, is likely to cause secondary pollution. In response to these problems, this paper carried out an exploratory research that can achieve indoor and outdoor air purification device. According to the clean air volume of HEPA filter, the pressure of fan and the cross-sectional area of filter are determined, meanwhile, the air resistance of indoor and outdoor circulating air purifier, the effect of air blower on air purification efficiency and filter CADR are discussed in detail. According to the filtering characteristics of single-layer filter, a kind of indoor and outdoor circulating air purifier-single-layer filter type air purifier was designed. Among the air purifier, the PLC control circuit is used to achieve single-layer filter air purifier filter automatically replace.
\end{abstract}

\section{Introduction}

In the winter of 2012, air pollution in China was so severe that one-third of the Chinese territory was covered by fog and haze, and the continuously hazy weather in most big cities lasted for more than 30 days [1]. At the beginning of 2016, haze appeared in many places in China. During the severe smog period, many highways were blocked; the aircraft were grounded; the school was closed; patients with respiratory diseases increased significantly, and the normal production and living and physical health of the local residents have been severely damaged. The main component of haze is PM2.5. Research shows exposure to particulate matter less than $2.5 \mu \mathrm{m}$ in diameter (PM2.5) is associated with asthma and respiratory symptoms and etc [2].

It should be pointed out that the annual average concentration of PM2.5 standard set by the World Health Organization is less than $10 \mu \mathrm{g} / \mathrm{m} 3$, and the annual average concentration of $35 \mu \mathrm{g} / \mathrm{m} 3$ will greatly increase the incidence of haze and death. A recent Chinese case study concluded that the reduction in life expectancy of about 3 years may be expected from long-term exposure to an additional $100 \mu \mathrm{g} / \mathrm{m} 3$ of Total Suspended Particles (TSPs) [3]. The status quo of haze pollution in China has been extremely serious. The problem of haze pollution control has long been impeded.

There are several purification technologies in air purifiers such as HEPA(high efficiency particulate air filter), mineralization technology of ultra-light structure, negative ion technology, the photocatalyst technology, electrostatic dust collection, activated carbon adsorption technology and etc [4].

There are two main types of air purifiers on the market in China. The first air purifier usually uses the filter with activated carbon to adsorb the tiny particles. The working capacity of this kind of purifier is limited. The purifying efficiency of the harmful gas and the dust will gradually decrease with the use time until it is completely deactivated. At this moment, the pollutants will accumulate in the indoor and the filter net, which seriously threatens human health. In severe haze, the filter needs to be replaced every $0.5-3$ months, and each time the cost of more than $100 \mathrm{RMB}$, greatly increased the cost of late maintenance. The second air purifier uses high-voltage electrostatic to capture dust particles, but electrostatic dust removal technology can only remove particulate pollutants, cannot remove harmful gas pollutants. In addition, electrostatic dust collection has discharge electrode in which a direct current is applied [5], and the relevant high-voltage electrostatic generator, such as the discharge needles, need regular cleaning. The process is difficult to operate, and easily damaged, expensive, especially high voltage electrostatic technology will produce a large number of harmful ozone. At present, two types of air purifiers on the market are the circulation of the original indoor air, the circulation of indoor and outdoor air cannot be achieved, and the fresh air cannot be provided to the room.

\section{Methodology}

This paper designs an air purifier that can purify the outdoor air to the room and fresh air is supplied to the room by filtering outdoor air. The air pressure in the room is greater than the outdoor air pressure so that the air in the room passes through the gaps to the outdoor one-way circulation and prevents outdoor air backflow from bringing atmospheric pollutants into the room. In addition, the current air purifier filter on the market cannot be

Corresponding author: dut@smm.neu.edu.cn 
automatically replaced. Once used for some time, the filter will accumulate large amounts of dust and other pollutants in the absorption. If not timely replacement, it will be likely to cause secondary pollution. Therefore, this paper designed a single-layer filter air purifier that can automatically replace the filter through the PLC. Compared to the folded filter, this single-layer filter design will greatly improve the filter's life and the volume of dust of the same area. The price will be relatively decreased, while not causing secondary pollution of indoor air.

\subsection{HEPA filter}

HEPA filter efficiency for the 0.3 micron fine particles can reach $99.7 \%$. Its characteristic is the air can pass, but the tiny particles cannot pass. HEPA filter is the most effective smoke, dust, bacteria and other pollutants filter material. Therefore, this paper chose Spring CMI300 HEPA filter net as a filter material.

\subsection{Differential pressure gauge}

The Testo510 (shown in Figure 1) is a device for measuring differential pressure. It is commonly used for measuring differential pressure (such as measuring filter net permeability) and gas-liquid pressure,. The readings are accurate and reliable.

\subsection{Anemometer}

In this paper, Testo405-V1 anemometer is used to measure the outlet air velocity of the air purifier, and the clean air volume of the air purifier is obtained according to the outlet cross-sectional area. Testo405-V1 (shown in Figure 2) is a thermal anemometer. With a telescopic handle (up to $300 \mathrm{~mm}$ extension), it measures wind velocity, air volume and temperature. It is suitable for detecting ventilation ducts, vents or unsealed windows and can read out the number directly, responsive, easy to operate.

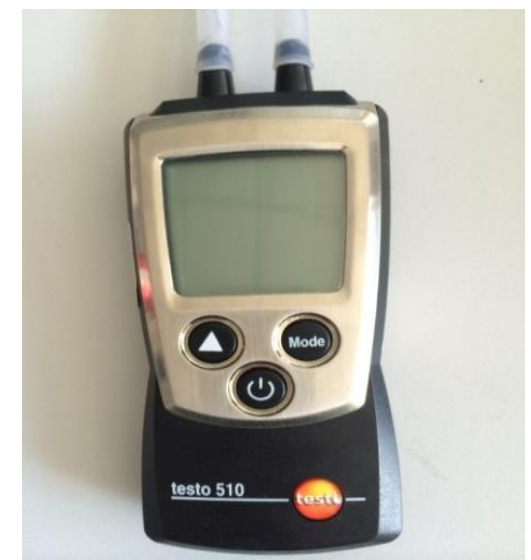

Figure 1. Differential pressure gauge(Testo510)

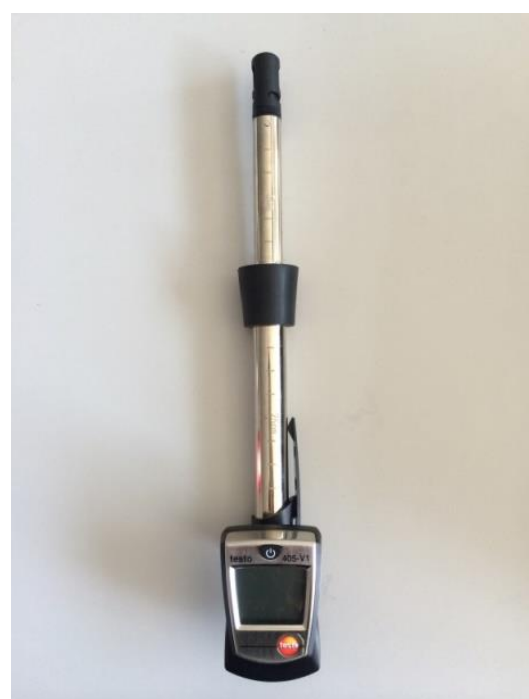

Figure 2. Anemometer(Testo405-V1)

\subsection{Air purifier}

In order to facilitate the study of the filter performance of the filter, the fan of Panasonic F-PDF35C air purifier was used as a new air purifier design of the fan, and the original filter net are replaced by the HEPA filter net we want to study. Then a series of performance parameters of the modified air purifier were tested to determine the PM2.5 filtration performance of our experimental raw HEPA filter. The Panasonic F-PDF35C air purifier (shown in Figure 3) with low, medium and high speed windshield design can achieve the expected wind speed.

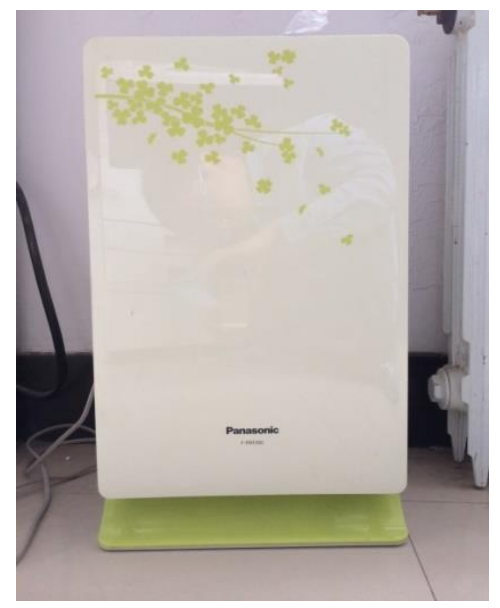

Figure 3. Air Purifier (Panasonic F-PDF35C)

\section{Results and discussion}

\subsection{Pressure analysis of fan}

Testo510-type pressure anemometer was used to test Panasonic F-PDF35C air purifier corresponding to the different levels of pressure, and that is the fan pressure under different wind velocity. When measuring, the Panasonic F-PDF35C air purifier does not have any HEPA filter installed. Open the air purifier power supply, respectively, in the low, medium and high three different 
wind speed levels, with a differential pressure test before and after the air purifier fan pressure. In order to reduce the experimental error, it was measured three times, and the final results take the average, the results are shown in Figure 4. It can be seen from Figure 4 that the differential pressures before and after the fan are $17 \mathrm{~Pa}, 60 \mathrm{~Pa}$ and $130 \mathrm{~Pa}$ respectively at low wind velocity, middle speed velocity and high wind velocity, which provides the wind resistance for the design of the air purifier in the later chapters of this paper.

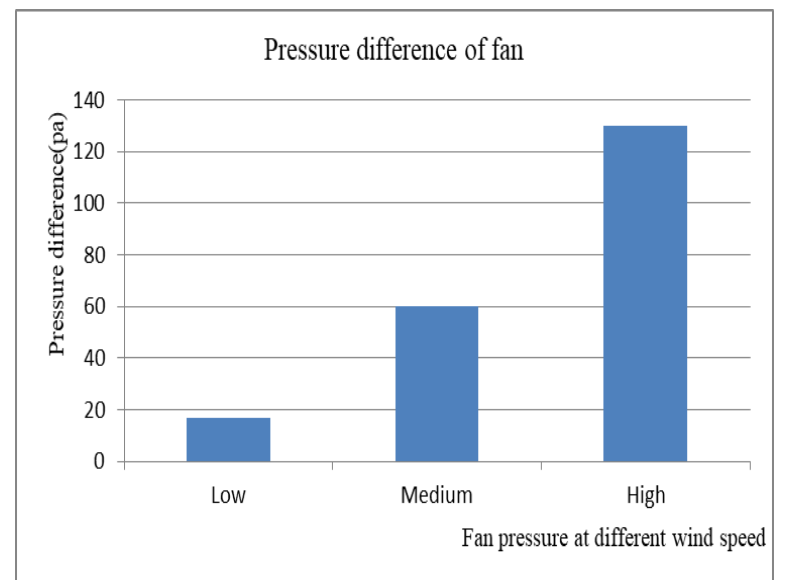

Figure 4. the pressure of fan at different velocities

\subsection{Filtration efficiency analysis}

In order to understand the filtration efficiency of the filter, the filtration efficiency of the PM2.5 in the aerosol of the environment under low, medium and high filtration velocities was tested. The specific experiment was carried out in a $60 \mathrm{~cm}^{*} 60 \mathrm{~cm} * 60 \mathrm{~cm}$ closed acrylic box. Lighting a cigarette, then using PM2.5 tester PM2.5 tests concentration in the acrylic box. When the PM2.5 concentration was $990 \mu \mathrm{g} / \mathrm{m} 3$, the air purifier was turned on and the initial concentration was set at $990 \mu \mathrm{g} / \mathrm{m} 3$. At this point, the outlet gas concentration of air purifier was regarded as the final concentration.

The filtration efficiency of the filter $(\eta)$ is defined as the ratio of the difference between the initial concentration $\mathrm{c} 1$ of the air purifier outlet and the final concentration $\mathrm{c} 2$ to the initial concentration value. The calculation formula is shown in the following formula (1):

$$
\eta=\frac{c_{1}-c_{2}}{c_{1}} \times 100 \%
$$

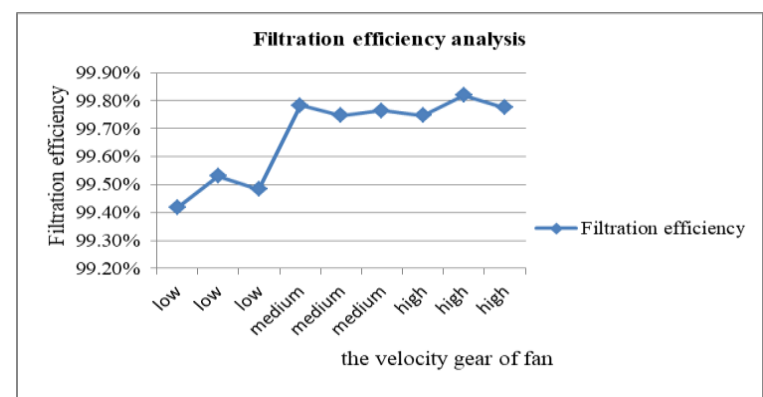

Figure 5. Filtration efficiency at different wind speed
The result of filtration efficiency analysis is shown in Figure 5. Regardless of the wind velocity of fan, the air purifier purification efficiency was always more than $99 \%$, so the wind velocity had little effect on purification efficiency of the Spring CMI300 HEPA filter, and Spring CMI300 HEPA filter was suitable for air purifier filter.

\subsection{CADR analysis}

Air purifier outlet size: length $\times$ width $=11 \mathrm{~cm} \times 7 \mathrm{~cm}$. The outlet cross-sectional area is $77 \mathrm{~cm} 2$, and wind speed of the outlet can be measured by Testo $405-\mathrm{V} 1$ anemometer. The amount of clean air (CADR) can be calculated based on the filtration velocity $(\mathrm{v})$ and the cross-sectional area (c). The calculation formula is shown in the following formula (2).

$$
C A D R=v \times \mathrm{c}
$$

According to formula (2) to calculate the amount of clean air filter, the result is shown in Figure 6.

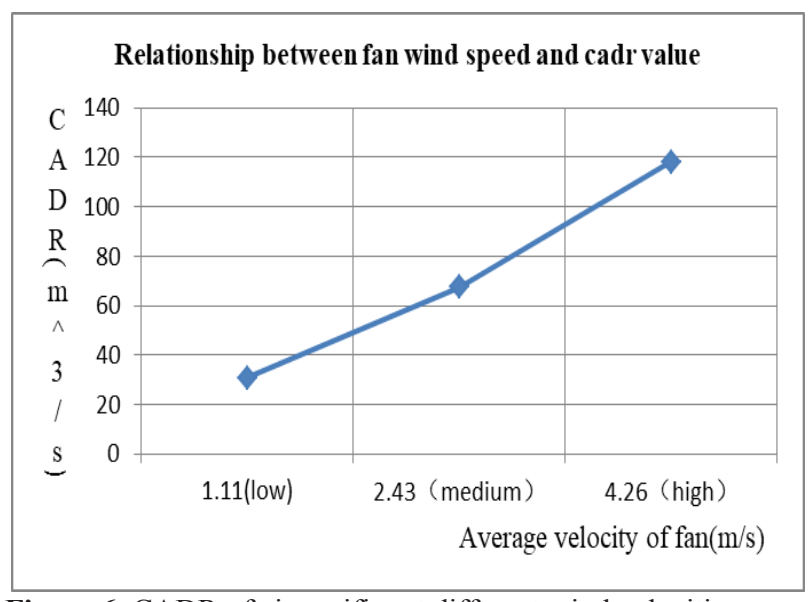

Figure 6. CADR of air purifier at different wind velocities

\section{Model device}

As is shown in Figure 7, the air purifier introduces the outdoor air into the room through a fan. After the filtration by the HEPA filter, clean purified air outside the room is introduced into the room to circulate indoor and outdoor air. When accumulative pollutants on the air purifier filter gradually increased, the purification effect will gradually decline. According to the consumption guideline of GB and AQSIQ, by reducing the CCM value (When the CADR value changes to $50 \%$ of the initial CADR value, the total weight of the purified pollutants in a unit of $\mathrm{mg}$ is the CCM value), CCM value can be converted into the average daily capacity. Then the number of filter days which is the filter replacement cycle can be calculated. As is shown in Figure 8 and Figure 9, when the filter net reaches the service life, the PLC control system starts the motor through the timer and automatically drives the filter net to be replaced. The process that filter net automatically changes the filter net is shown in Figure 10. 


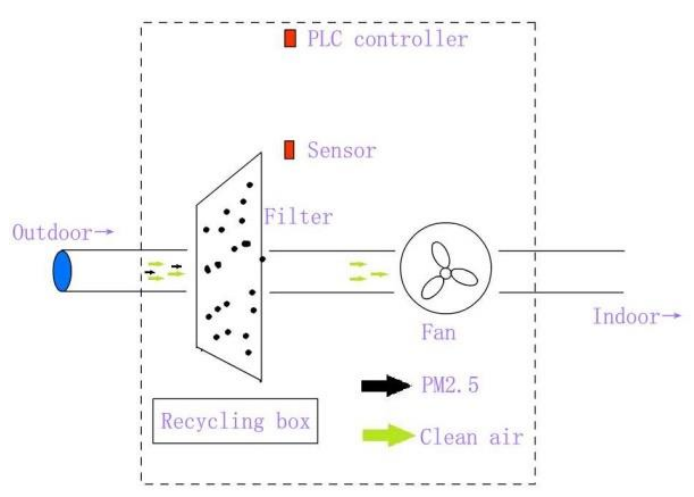

Figure 7. Adsorption process of filter net

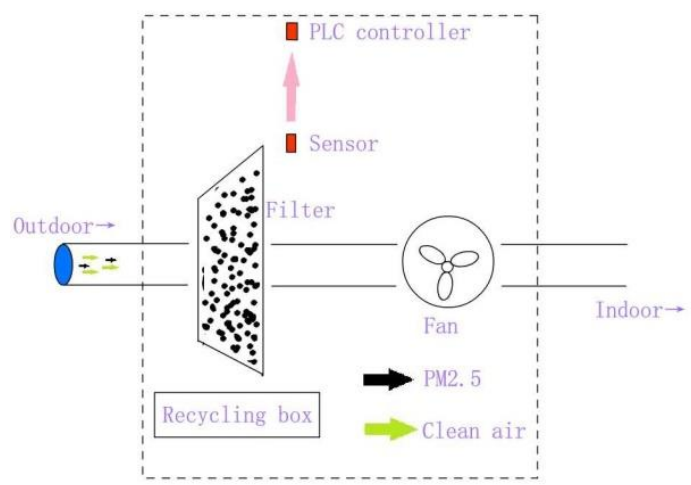

Figure 8. The filter is about to be full

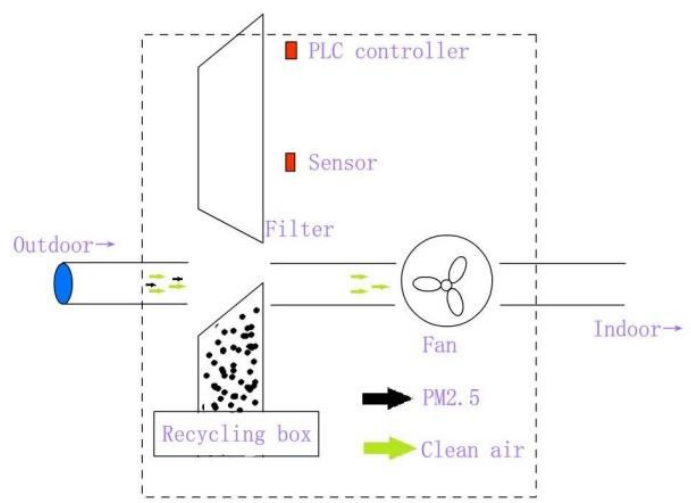

Figure 9. Automatic replacement starts

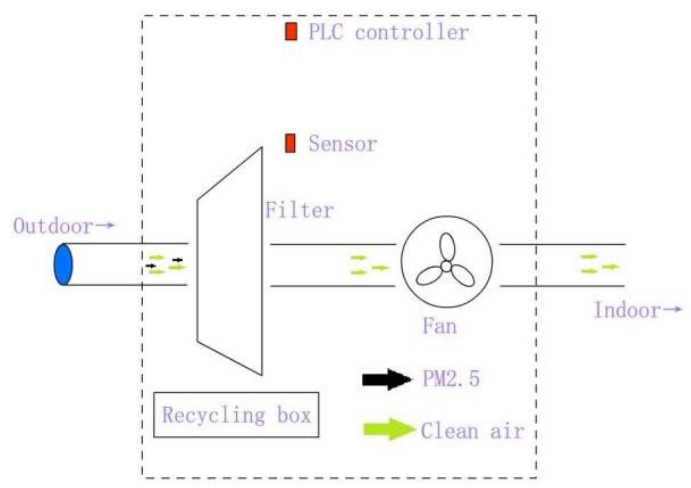

Figure 10. Automatic replacement ends

\section{PLC control system}

Programmable logic controller (PLC) is a basic and essential element in the engineering of industrial automation [6]. It uses a programmable memory to store instructions and execute functions including logical control, sequencing, timing, counting and arithmetic [7]. In this paper, the control system uses the Siemens S7-200 PLC with good reliability and scalability as the controller to design the LAD circuit. As is shown in Figure 11, I0.0 is the button, $\mathrm{C} 0, \mathrm{C} 1, \mathrm{C} 2$ is a counter, $\mathrm{SM} 0.5$ is a pulse signal, and Q0.0 is to replace the filter motor. The time base of this LAD circuit is one hour. VW is defined as replacement cycle in a unit of hour. When the filter reaches the end of its service life, Network 1 opens the way for the motor to start and automatic filter replacement is complete. Therefore, the problem of secondary air pollution has been solved.

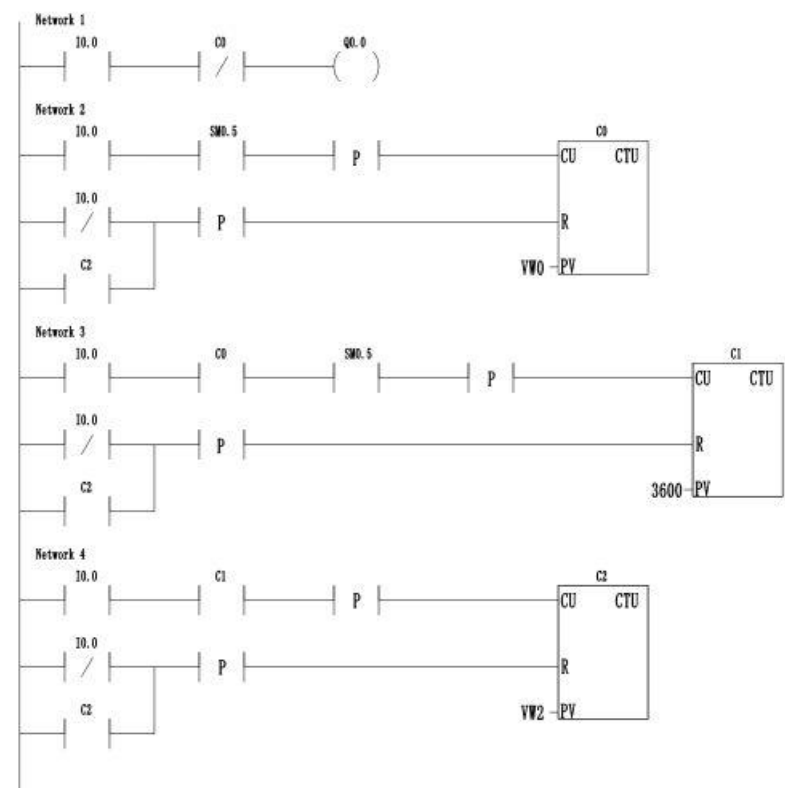

Figure 11. PLC automatic control system works

\section{Conclusions}

The current existing air purifier purifies the original air in the room, and cannot be indoor and outdoor air circulation and provide fresh air to the room. So, in this paper, the design of indoor and outdoor air purifier was studied. To sum up, first, the wind resistance, filtration speed, clean air volume and dust holding capacity of each filter were analyzed to find out the most suitable filter net for indoor and outdoor air purifiers. Then according to the characteristics of a single filter net, a kind of indoor and outdoor air purifier was designed.

As the air purifier filter cannot be automatically replaced in time, this paper uses the Siemens S7-200 series PLC programming software to achieve the air purifier replacement filter automatically. If system need to change the filter net replacement cycle, changing the value of VW can achieve it. Automatic replacement filter net of air purifier control system is only a typical application in the intelligent control model. With the rapid development of science and technology, the information technology with computer technology, communication technology and software technology as 
the core has achieved more rapid development. All kinds of equipment can be combined with this technology. Wide range of applications and computing systems, intelligent systems will gradually change the lives of humans.

\section{Acknowledgments}

The authors gratefully acknowledge the financial support of Innovation and entrepreneurship college of Northeastern University (Grants 170045) and Natural Science Foundation of China (Grants 51406029 and 51474067) and the guide of Professor T Du and Professor L Y Liu.

\section{References}

1. Chao Ye, Ruishan Chen, Mingxing Chen 2015 The impacts of Chinese Nian culture on air pollution, Sustainable Cities and Society, v 31, p 26-36, May 2017

2. Mimura, Tatsuya, Ichinose, Takamichi, Yamagami and Satoru 2014 Airborne particulate matter (PM2.5) and the prevalence of allergic conjunctivitis in Japan, Science of the Total Environment, v 487, n 1, p 493499, July 15, 2014

3. Y. Chen, A. Ebenstein, M. Greenstone, H. Li

Evidence on the impact of sustained exposure to air pollution on life expectancy from China's Huai River policy

Proc. Natl. Acad. Sci. U. S. A., 110 (2013), pp. 12936-12941, 10.1073/pnas.1300018110

4. Yunhan Wang, Hao Wang, Changzhong Zhao, Yi Zhang 2015 Advanced Materials Research, v 10921093, 1025-8, 2015

5. SEKIKAWA T, ABE T, OGASAWARA M, SHIBAYAMA H 2016-61682LWO2016153046-A1; KR2017125968-A

6. M Mahadi, NA Mohd Ami, M Ab Rahim, MS Abdul Majid 2015 Applied Mechanics and Materials, v 786, 367-71, 2015

DOI: $10.4028 /$ www.scientific.net/AMM.786.367

7. C. D. Johnson, Process Control Instrumentation Technology, Prentice Hall., Upper SaddleRiver, New Jersey, 2006. 